\title{
A generating function for Hermite polynomials in connection with Euclidean Landau levels
}

\author{
Zouhaïr MOUAYN \\ Department of Mathematics, Faculty of Sciences and Technics (M'Ghila), Sultan Moulay Slimane \\ University, BP 523, Béni Mellal, Morocco \\ (e-mail: mouayn@gmail.com)
}

\begin{abstract}
We have formulated a generating function for the Hermite polynomials by comparing two expressions of the same coherent states attached to planar Landau levels. A first expression is obtained by generalizing the canonical coherent states when written as series expansion in the basis of number states. While the second expression is established by following a construction based on group representation theory.
\end{abstract}

\section{Introduction}

An important class of orthogonal polynomial encountered in the applications, especially in mathematical physics, consists of the Hermite polynomials $H_{n}(\xi)$ which can be defined by the Rodriguez formula as

$$
H_{n}(\xi)=(-1)^{n} e^{\xi^{2}} \frac{d^{n}}{d \xi^{n}}\left(e^{-\xi^{2}}\right), \quad n=0,1,2, \cdots
$$

According to (1.1), the first few Hermite polynomials are

$$
H_{0}(\xi)=1, \quad H_{1}(\xi)=2 \xi, \quad H_{2}(\xi)=4 \xi^{2}-2, \cdots,
$$

and in general,

$$
H_{n}(\xi)=\sum_{k=0}^{[n / 2]} \frac{(-1)^{k} n !}{k !(n-2 k) !}(2 \xi)^{n-2 k}
$$

where $[\eta]$ denotes the largest integer $\leq \eta$. These polynomials multiplied by the constant factor $1 / n$ ! are the coefficients in the expansion

$$
\exp \left(2 \xi \tau-\tau^{2}\right)=\sum_{n=0}^{+\infty} \frac{H_{n}(\xi)}{n !} \tau^{n}
$$

called the generating function of the Hermite polynomials [1]. 
Here, our aim is to draw attention to an interesting relation satisfied by the Hermite polynomials, which arise when comparing two expressions of the same coherent states attached to planar Landau levels. The relation we propose here is a kind of generalization of (1.4) involving the polynomials

$$
L_{m}^{(k)}(x):=\sum_{j=\max (0,-k)}(-1)^{j} \frac{(k+m) !}{j !(m-k) !(k+j) !} x^{j}
$$

which correspond to the usual Laguerre polynomial for $k \geq 0$. However, equation (1.5) is valid for negative value of $k$ [17, p. 98]. Precisely, we establish the following formula:

Theorem 1.1. Let $m=0,1,2, \cdots, \beta>0$ and $a, b \in \mathbb{R}$. Then,

$$
\begin{aligned}
\sum_{k=-m}^{+\infty} \frac{(\sqrt{\beta}(a+i b))^{k}}{2^{k}(k+m) !} & L_{m}^{(k)}\left(\frac{\beta}{2}\left(a^{2}+b^{2}\right)\right) H_{k+m}(\xi) \\
& =\frac{1}{m !} \exp \left(-\frac{\beta}{4}(a-i b)^{2}+\xi(a-i b) \sqrt{\beta}\right) H_{m}(\xi-a \sqrt{\beta}) .
\end{aligned}
$$

Note that for $m=0, b=0$ and $a=2 \tau \beta^{-1 / 2}$, the relation (1.6) reduces to (1.4). I didn't find formula (1.6) in books dealing with special functions and orthogonal polynomials but after sending this manuscript to many colleagues I have received in response a letter [2] from Professor Mourad ISMAIL who has established a direct proof for this formula (1.6). I would like to thank him very much for reading the manuscript and for his interest on this work.

This paper is organized as follows. In Section 2, we recall some facts on the canonical coherent states of the quantum harmonic oscillator as well as two types of their generalization we will be dealing with. In Section 3, we summarize some needed tools on the planar Landau levels. In Section 4, we construct coherent states via a group representation theory. In Section 5, we rederive these coherent states via a series expansion.

\section{The canonical coherent states}

The first model of coherent states was the 'nonspreading wavepacket' of the harmonic oscillator, which have been constructed by Schrödinger [3]. In suitable units, wave functions of these states can be written as

$$
\Phi_{\mathfrak{z}}(\tilde{\xi}):=\langle\xi \mid \mathfrak{z}\rangle=\pi^{-\frac{1}{2}} \exp \left(-\frac{1}{2} \xi^{2}+\sqrt{2} \mathfrak{\xi} \mathfrak{z}-\frac{1}{2} \mathfrak{z}^{2}-\frac{1}{2}|\mathfrak{z}|^{2}\right), \xi \in \mathbb{R},
$$

where $\mathfrak{z} \in \mathbb{C}$ determines the mean values of coordinate $\widehat{x}$ and momentum $\hat{p}$ according to $\langle\widehat{x}\rangle:=\left\langle\Phi_{\mathfrak{z}}, x \Phi_{\mathfrak{z}}\right\rangle=\sqrt{2} \Re \mathfrak{z}$ and $\langle\hat{p}\rangle:=\left\langle\Phi_{\mathfrak{z}}, p \Phi_{\mathfrak{z}}\right\rangle=\sqrt{2} \Im_{\mathfrak{z}}$. The variances $\sigma_{x}=\left\langle\widehat{x}^{2}\right\rangle-\langle\widehat{x}\rangle^{2}=$ $\frac{1}{2}$ and $\sigma_{p}=\left\langle\hat{p}^{2}\right\rangle-\langle\hat{p}\rangle^{2}=\frac{1}{2}$ have equal values, so their product assumes the minimal value permitted by the Heisenberg uncertainty relation.

The coherent state $\Phi_{\mathfrak{z}}$ have been also obtained by Feymann [4] and Glauber [5] from the vacuum state $|0\rangle$ by means of the unitary displacement operator $\exp \left(\mathfrak{z} A^{*}-\overline{\mathfrak{z}} A\right)$ as

$$
\Phi_{\mathfrak{z}}=\exp \left(\mathfrak{z} A^{*}-\overline{\mathfrak{z}} A\right)|0\rangle,
$$


where $A$ and $A^{*}$ are annihilation and creation operators defined by

$$
A=\frac{1}{\sqrt{2}}(\widehat{x}+i \widehat{p}), \quad A^{*}=\frac{1}{\sqrt{2}}(\widehat{x}-i \widehat{p}) .
$$

It was Iwata [6] who used the well known expansion over the Fock basis $|n\rangle$ to give an expression of $\Phi_{\mathfrak{z}}$ as

$$
\Phi_{\mathfrak{z}}=e^{-\frac{1}{2}|\mathfrak{z}|^{2}} \sum_{n=0}^{+\infty} \frac{\mathfrak{z}^{n}}{\sqrt{n !}}|n\rangle .
$$

Actually, various generalizations of coherent states have been proposed. Here, we shall focus on two of them: (i) a group-theoretic generalization "à la Perelomov"of (2.2) in which coherent states are produced by the action $T_{g}$ of the group element $g \in G$ on a reference state $\phi_{0}$ in a representation Hilbert space $\mathcal{H}$ as ([[]]):

$$
\widetilde{\Phi}_{\mathfrak{g}}=T_{g}\left[\phi_{0}\right],
$$

(i) a generalization "à la Iwata" of (2.4), by choosing different set of coefficients $\left\{c_{n}(\mathfrak{z})\right\}$ and functions $\left\{\varphi_{n}\right\}$ satisfying suitable conditions as

$$
\widetilde{\Phi}_{\mathfrak{z}}=\sum_{n} c_{n}(\mathfrak{z})\left|\varphi_{n}\right\rangle .
$$

For an overview of all aspect of the theory of coherent states we refer to the survey of V.V. Dodonov [8].

\section{Landau levels on $\mathbb{R}^{2}$}

The Hamiltonian of a single electron, moving on a two-dimensional plane subject to a uniform magnetic field along the $z$-direction, is given by

$$
H_{\beta}:=\frac{1}{2}\left(i \partial_{x}-\frac{\beta}{2} y\right)^{2}+\frac{1}{2}\left(i \partial_{y}+\frac{\beta}{2} x\right)^{2},
$$

where $\beta>0$ is proportional to the field strength. $H_{\beta}$ is an unbounded symmetric operator on $C_{0}^{\infty}\left(\mathbb{R}^{2}\right)$ and is an essentially self-adjoint operator in $L^{2}\left(\mathbb{R}^{2}, d \mu(x, y)\right), d \mu$ being the Lebesgue measure on $\mathbb{R}^{2}$. The spectrum of $H_{\beta}$ consists of eigenvalues of infinite multiplicity (Landau levels) of the form

$$
\epsilon_{m}^{\beta}:=\left(m+\frac{1}{2}\right) \beta, \quad m=0,1,2, \cdots .
$$

We let $\mathcal{A}_{m, \beta}\left(\mathbb{R}^{2}\right)$ denote the eigensubspace of the operator $H_{\beta}$, which corresponds to the eigenvalue $\epsilon_{m}^{\beta}$ in (3.2) as

$$
\mathcal{A}_{m, \beta}\left(\mathbb{R}^{2}\right):=\left\{\varphi \in L^{2}\left(\mathbb{R}^{2}\right), H_{\beta} \varphi=\epsilon_{m}^{B} \varphi\right\} .
$$


Our next goal is to consider a basis of the subspace in (3.3). For $(x, y) \in \mathbb{R}^{2}$, and $k=$ $-m,-m+1, \cdots$, set

$$
\varphi_{k}^{\beta, m}(x, y):=c_{\beta, m, k}\left(\sqrt{\frac{\beta}{2}}(x+i y)\right)^{k} e^{-\frac{\beta}{4}\left(x^{2}+y^{2}\right)} L_{m}^{(k)}\left(\frac{\beta}{2}\left(x^{2}+y^{2}\right)\right),
$$

where

$$
c_{\beta, m, k}=\left(\frac{m ! \beta}{2 \pi(k+m) !}\right)^{\frac{1}{2}} .
$$

The functions $\varphi_{k}^{\beta, m}, k=-m,-m+1, \cdots$, constitute an orthonormal basis of the Hilbert space $\mathcal{A}_{m, \beta}\left(\mathbb{R}^{2}\right)$ whose reproducing kernel is given by

$$
K_{m, \beta}\left((x, y),\left(x^{\prime}, y^{\prime}\right)\right):=\sum_{k=-m}^{+\infty} \varphi_{k}^{\beta, m}(x, y) \overline{\varphi_{k}^{\beta, m}\left(x^{\prime}, y^{\prime}\right)} .
$$

Explicitly, we have that ([9, p. 1058]):

$$
K_{m, \beta}\left(\mathbf{r}, \mathbf{r}^{\prime}\right)=\frac{\beta}{2 \pi} \exp \left(-\frac{i \beta}{2} \mathbf{r} \wedge \mathbf{r}^{\prime}-\frac{\beta}{4}\left\|\mathbf{r}-\mathbf{r}^{\prime}\right\|^{2}\right) L_{m}^{(0)}\left(\frac{\beta}{2}\left\|\mathbf{r}-\mathbf{r}^{\prime}\right\|^{2}\right) .
$$

where $\mathbf{r}=(x, y), \mathbf{r}^{\prime}=\left(x^{\prime}, y^{\prime}\right) \in \mathbb{R}^{2}$ and $\mathbf{r} \wedge \mathbf{r}^{\prime}=x y^{\prime}-x^{\prime} y$.

For more information on Landau levels we refer the reader to [10].

\section{Perelomov's coherent states}

As pointed out in Section 2, various generalizations of the notion of coherent states have been proposed. Here, for our purpose, we adopt the so called Prelomov's approach based on a group-theoretic formalism [7].

For this, let $\mathbb{H}_{1}$ be the Heisenberg group (of degree 1) i.e. the Lie group whose underlying manifold is $\mathbb{R}^{3}$ with coordinates $(x, y, t)$ and whose group law is

$$
(x, y, t) \cdot\left(x^{\prime}, y^{\prime}, s\right)=\left(x+x^{\prime}, y+y^{\prime}, t+s+\frac{1}{2}\left(x y^{\prime}-x^{\prime} y\right)\right) .
$$

It is standard that Lebesgue measure on $\mathbb{R}^{3}$ is bi-invariant Haar measure on $\mathbb{H}_{1}$. The continuous unitary irreducible representations of $\mathbb{H}_{1}$ are well known [11, p. 37]. Here, we will be concerned with a representation of $\mathbb{H}_{1}$ on the Hilbert space $L^{2}(\mathbb{R}, d \xi)$ by shift and multiplication operators [12, §1.1]:

$$
T_{\beta}(x, y, t)[\psi](\xi):=\exp i\left(\beta t-\sqrt{\beta} y \xi+\frac{\beta}{2} x y\right) \psi(\xi-\sqrt{\beta} x)
$$

for $(x, y, t) \in \mathbb{H}_{1}, \beta>0, \psi \in L^{2}(\mathbb{R}, d \xi)$ and $\xi \in \mathbb{R}$, called the Schrödinger representation. Proposition 4.1.The Schrödinger representation $T_{\beta}$ in (4.2) satisfies the equality:

$$
\int_{\mathbb{R}^{2}}\left\langle\psi_{1}, T_{\beta}(x, y, 0)\left[\phi_{1}\right]\right\rangle\left\langle T_{\beta}(x, y, 0)\left[\phi_{2}\right], \psi_{2}\right\rangle d \mu(x, y)=\left\langle\psi_{1}, \psi_{2}\right\rangle\left\langle\phi_{1}, \phi_{2}\right\rangle
$$

for all $\psi_{1}, \psi_{2} \in L^{2}(\mathbb{R}, d \xi)$ and $\phi_{1}, \phi_{2} \in L^{2}(\mathbb{R}, d \xi)$. 
Proof. By [13], the unitary irreducible representation $T_{\beta}$ in (4.2) is square integrable modulo: the center $\mathbb{R}$ of $\mathbb{H}_{1}$ and the Borelian section $\sigma_{0}$ defined from the coset space $\mathbb{H}_{1} / \mathbb{R}=\mathbb{R}^{2}$ into $\mathbb{H}_{1}$ by $\sigma_{0}(x, y):=(x, y, 0)$. Next, by theorem [14] there exists operator $\delta$ in $L^{2}(\mathbb{R}, d \xi)$ selfadjoint, positive and semi-invariant such that

$$
\int_{\mathbb{R}^{2}}\left\langle\psi_{1}, T_{\beta}(x, y, 0)\left[\phi_{1}\right]\right\rangle\left\langle T_{\beta}(x, y, 0)\left[\phi_{2}\right], \psi_{2}\right\rangle d \mu(x, y)=\left\langle\psi_{1}, \psi_{2}\right\rangle\left\langle\delta^{\frac{1}{2}} \phi_{1}, \delta^{\frac{1}{2}} \phi_{2}\right\rangle
$$

for all $\psi_{1}, \psi_{2} \in L^{2}(\mathbb{R}, d \xi)$ and $\phi_{1}, \phi_{2} \in \operatorname{Dom}\left(\delta^{\frac{1}{2}}\right)$. Here, the group $\mathbb{H}_{1}$ is unimodular therefore the operator $\delta$ is the identity ([14, p. 215]).

Definition 4.1. Let $m=0,1, \cdots$, and $\beta>0$. The Perelomov's coherent states attached to the Landau level $\epsilon_{m}^{\beta}$ in (3.2) and labeled by points $(x, y)$ of the coset space $\mathbb{R}^{2}$ are defined by

$$
\Phi_{(x, y), \beta, m}:=T_{\beta}\left(\sigma_{0}(x, y)\right)\left[\phi_{m}\right] .
$$

where the reference state $\phi_{m}$ is given by the Gaussian-Hermite function

$$
\phi_{m}(\xi):=\left(\sqrt{\pi} 2^{m} m !\right)^{-\frac{1}{2}} e^{-\frac{1}{2} \xi^{2}} H_{m}(\xi), \xi \in \mathbb{R} .
$$

Their wave functions are given by

$$
\Phi_{(x, y), \beta, m}(\xi)=\left(\sqrt{\pi} 2^{m} m !\right)^{-\frac{1}{2}} e^{-i \sqrt{\beta} \xi y+i \frac{\beta}{2} x y-\frac{1}{2}(\xi-\sqrt{\beta} x)^{2}} H_{m}(\xi-\sqrt{\beta} x) .
$$

Proposition 4.2. The unity of the Hilbert space $L^{2}(\mathbb{R}, d \xi)$ is solved as

$$
\mathbf{1}_{L^{2}(\mathbb{R}, d \xi)}=\int_{\mathbb{R}^{2}} d \mu(x, y)|(x, y), \beta, m><m, \beta,(x, y)|
$$

where the ket vector $\mid(x, y), \beta, m>\equiv \Phi_{(x, y), \beta, m}$.

Proof. The coherent states in (4.5) are completely justified by the square integrability of the unitary irreducible representations $T_{\beta}$ modulo the subgroup $\mathbb{R}$ and the section $\sigma_{0}$. In this context Eq. (4.3) reads

$$
\int_{\mathbb{R}^{2}}\left\langle\psi, \Phi_{(x, y), \beta, m}>\left\langle\Phi_{(x, y), \beta, m}, \psi\right\rangle d \mu(x, y)=\langle\psi, \psi\rangle, \quad \psi \in L^{2}(\mathbb{R}, d \xi) .\right.
$$

The square integrability of the representation $T_{\beta} \bmod \left(\sigma_{0}, \mathbb{R}\right)$ can be reformulated as a resolution of the identity. Indeed, denoting $\Phi_{(x, y), \beta, m}=\mid(x, y), B, m>$, one can use the Dirac's bra-ket notation to write (4.4) in the form (4.8). 


\section{$5 \quad$ Iwata's coherent states}

In this section, we present a generalization of the coherent states as given by Iwata's series (2.4) according to the procedure in [15].

Let $(X, v)$ be a measure space and let $\mathcal{A} \subset L^{2}(X, v)$ be a closed subspace of infinite dimension. Let $\left\{f_{n}\right\}_{n=1}^{\infty}$ be an orthogonal basis of $\mathcal{A}$ satisfying, for arbitrary $u \in X$,

$$
\omega(u):=\sum_{n=1}^{\infty} \rho_{n}^{-1}\left|f_{n}(u)\right|^{2}<+\infty,
$$

where $\rho_{n}:=\left\|f_{n}\right\|_{L^{2}(X)}^{2}$. Define

$$
K(u, v):=\sum_{n=1}^{\infty} \rho_{n}^{-1} f_{n}(u) \overline{f_{n}(v)}, \quad u, v \in X
$$

Then, $K(u, v)$ is a reproducing kernel, $\mathcal{A}$ is the corresponding reproducing kernel Hilbert space and $\omega(u)=K(u, u), u \in X$.

Definition 5.1. Let $\mathcal{H}$ be a Hilbert space with $\operatorname{dim} \mathcal{H}=\infty$ and $\left\{\phi_{n}\right\}_{n=1}^{\infty}$ be an orthonormal basis of $\mathcal{H}$. Therefore, for $x \in X$, define

$$
\mid u>:=(\omega(u))^{-\frac{1}{2}} \sum_{n=1}^{\infty} \frac{f_{n}(u)}{\sqrt{\rho_{n}}} \phi_{n} .
$$

The vectors $(\mid u>)_{u \in X}$ will be called coherent states of Iwata type.

The choice of the Hilbert space $\mathcal{H}$ defines a quantization of the set $X=\{u\}$ by the coherent states $\mid u>$, via the inclusion map : $u \rightarrow \mid u>$ from $X$ into $\mathcal{H}$. By definition, it is straightforward to show that $\langle u \mid u\rangle=1$. This also leads to the following definition:

Definition 5.2. The isometric map $W: \mathcal{H} \rightarrow \mathcal{A} \subset L^{2}(X, v)$ defined by $W[\phi](u):=(\omega(u))^{\frac{1}{2}}<$ $u \mid \phi>$ is called the coherent state transform associated with the set of coherent states $(\mid u>)_{u \in X}$.

Thus, for $\phi, \psi \in \mathcal{H}$, we have

$$
<\phi\left|\psi>_{\mathcal{H}}=<W[\phi]\right| W[\psi]>_{L^{2}(X)}=\int_{X} d v(u) \omega(u)<\phi|u><u| \psi>
$$

and thereby we have a resolution of the identity

$$
\mathbf{1}_{\mathcal{H}}=\int_{X} d v(u) \omega(u)|u><u|
$$

where $\omega(u)$ appears as a weight function. 
Definition 5.3. For each Landau level $\epsilon_{m}^{\beta}$ in (3.2) define a set of coherent states according to formula (5.3) as

$$
\mid(x, y), \beta, m>:=\left(K_{\beta, m}((x, y),(x, y))\right)^{-\frac{1}{2}} \sum_{n=0}^{+\infty} \frac{\varphi_{n}^{\beta, m}(x, y)}{\sqrt{\rho_{n}^{\beta, m}}} \phi_{n},
$$

where $K_{\beta, m}(. .$.$) is the reproducing kernel given in (3.7), \varphi_{n}^{\beta, m}(x, y)$ are the functions given in (3.4) and

$$
\phi_{n}(\xi)=\left(\sqrt{\pi} 2^{n} n !\right)^{-\frac{1}{2}} e^{-\frac{1}{2} \xi^{2}} H_{n}(\xi) .
$$

It is well known [16] that the functions $\phi_{n}(\xi), n=0,1, \cdots$, constitute an orthonormal basis of the Hilbert space $L^{2}(\mathbb{R}, d \xi)$. The latter carries the constructed coherent states in (5.6).

Proposition 5.1. The wave functions of the states (5.6) can be expressed in a closed form by the expression in (4.7) . i.e., the equality

$$
<\xi \mid(x, y), \beta, m>=\Phi_{(x, y), \beta, m}(\xi) .
$$

holds for any $\xi \in \mathbb{R}$.

Proof. According to Eq. (5.6), we start by writing

$$
\begin{aligned}
& <\xi \mid(x, y), \beta, m>:=\left(\frac{\beta}{2 \pi}\right)^{-\frac{1}{2}} \sum_{n=0}^{+\infty} \varphi_{n}^{\beta, m}(x, y) \phi_{n}(\xi) \\
& \quad=e^{-\frac{\beta}{4}\left(x^{2}+y^{2}\right)} \sum_{n=0}^{+\infty} \sqrt{\frac{m !}{n !}}\left(\sqrt{\frac{\beta}{2}}\right)^{n-m}(x+i y)^{n-m} L_{m}^{(n-m)}\left(\frac{\beta\left(x^{2}+y^{2}\right)}{2}\right) \phi_{n}(\xi)
\end{aligned}
$$

But since $n$ takes values from 0 to $+\infty$, we have to take care when $0 \leq n<m$. In this case we make use of Eq. (1.5) or [17, p. 98] to write

$$
L_{m}^{(-p)}(w)=(-w)^{p} \frac{(m-p) !}{m !} L_{m-l}^{(p)}(w), \quad 1 \leq p \leq m
$$

for $p=m-n$ to write the Laguerre polynomial with upper index $n-m<0$ as

$$
L_{m}^{(n-m)}\left(\frac{\beta\left(x^{2}+y^{2}\right)}{2}\right)=\left(-\frac{\beta}{2}\left(x^{2}+y^{2}\right)\right)^{m-n} \frac{n !}{m !} L_{n}^{(m-n)}\left(\frac{\beta\left(x^{2}+y^{2}\right)}{2}\right) .
$$

Next we make use of the identity [1, p. 838]

$$
\int_{-\infty}^{+\infty} e^{-u^{2}} H_{s}(u+\alpha) H_{l}(u+\gamma) d u=2^{l} \sqrt{\pi} s ! \gamma^{l-s} L_{s}^{(l-s)}(-2 \alpha \gamma), \quad s \leq l,
$$

for

$$
-2 \alpha \gamma=\frac{\beta}{2}\left(x^{2}+y^{2}\right), \alpha=-\frac{\sqrt{\beta}}{2}(x-i y), \quad \gamma=\frac{\sqrt{\beta}}{2}(x+i y) .
$$


After calculations, the following identity holds for any $n=0,1,2, \cdots$,

$$
(x+i y)^{n-m} L_{m}^{(n-m)}\left(\frac{\beta}{2}\left(x^{2}+y^{2}\right)\right)=\frac{(\sqrt{\beta})^{m-n}}{2^{m} m ! \sqrt{\pi}} \int_{-\infty}^{+\infty} e^{-u^{2}} H_{m}(u+\alpha) H_{n}(u+\gamma) d u .
$$

Returning back to Eq. (5.8), it follows that

$$
\begin{aligned}
& <\xi \mid(x, y), \beta, m> \\
& =e^{-\frac{\beta}{4}\left(x^{2}+y^{2}\right)} \sum_{n=0}^{+\infty} \sqrt{\frac{m !}{n !}}\left(\frac{(\sqrt{2})^{n-m}}{2^{n} m ! \sqrt{\pi}} \int_{-\infty}^{+\infty} e^{-u^{2}} H_{m}(u+\alpha) H_{n}(u+\gamma) d u\right) \phi_{n}(\xi) \\
& =\frac{e^{-\frac{\beta}{4}\left(x^{2}+y^{2}\right)}}{(\sqrt{2})^{m} \sqrt{\pi m !}} \sum_{n=0}^{+\infty} \frac{1}{\sqrt{n !}(\sqrt{2})^{n}}\left(\int_{-\infty}^{+\infty} e^{-u^{2}} H_{m}(u+\alpha) H_{n}(u+\gamma) d u\right) \phi_{n}(\xi) \text {. }
\end{aligned}
$$

So, we have to consider the integral

$$
I_{m, n}=\int_{-\infty}^{+\infty} e^{-u^{2}} H_{m}(u+\alpha) H_{n}(u+\gamma) d u
$$

By the change of variable: $u \rightarrow u-\gamma$, we obtain that

$$
I_{m, n}=\int_{-\infty}^{+\infty} \exp \left(-u^{2}+2 u \gamma-\gamma^{2}\right) H_{m}(u+\alpha-\gamma) H_{n}(u) d u
$$

But, since

$$
\alpha-\gamma=-\frac{\sqrt{\beta}}{2}(x-i y)-\frac{\sqrt{\beta}}{2}(x+i y)=-\sqrt{\beta} x,
$$

we get successively

$$
\begin{aligned}
I_{m, n} & =\int_{-\infty}^{+\infty} \exp \left(-u^{2}+2 u \gamma-\gamma^{2}\right) H_{m}(u-\sqrt{\beta} x) H_{n}(u) d u \\
& =\int_{-\infty}^{+\infty} e^{-u^{2}+2 u \frac{\sqrt{\beta}}{2}(x+i y)-\left(\frac{\sqrt{\beta}}{2}(x+i y)\right)^{2}} H_{m}(u-\sqrt{\beta} x) H_{n}(u) d u \\
& =\int_{-\infty}^{+\infty} e^{i \sqrt{\beta} u y-i \frac{\beta}{2} x y-\frac{\beta}{4} x^{2}-u^{2}+u \sqrt{\beta} x} e^{\frac{\beta}{4} y^{2}} H_{m}(u-\sqrt{\beta} x) H_{n}(u) d u
\end{aligned}
$$


So that

$$
\begin{aligned}
& <\xi \mid(x, y), \beta, m> \\
& =\frac{(\sqrt{2})^{-m}}{\sqrt{m ! \pi}} \sum_{n=0}^{+\infty} \frac{1}{\sqrt{n !}}\left(\frac{1}{(\sqrt{2})^{n}} \int_{-\infty}^{+\infty} e^{-u^{2}} H_{m}(u+\alpha) H_{n}(u+\gamma) d u\right) \phi_{n}(\xi) \\
& =\frac{1}{(\sqrt{2})^{m} \sqrt{\pi} \sqrt{m !}} \sum_{n=0}^{+\infty} \frac{1}{\sqrt{n !}} \frac{\phi_{n}(\xi)}{(\sqrt{2})^{n}} \\
& \quad \times \int_{-\infty}^{+\infty} e^{-\frac{1}{2} u^{2}} e^{i \sqrt{\beta} u y-i \frac{\beta}{2} x y-\frac{\beta}{2} x^{2}-\frac{1}{2} u^{2}+u \sqrt{\beta} x} H_{m}(u-\sqrt{\beta} x) H_{n}(u) d u .
\end{aligned}
$$

Eq. (5.23) can also be written as

$$
\begin{aligned}
<\xi \mid(x, y), \beta, m>= & \sum_{n=0}^{+\infty} \phi_{n}(\xi) \\
& \int_{-\infty}^{+\infty} d u\left(\sqrt{\pi} 2^{n} n !\right)^{-\frac{1}{2}} e^{-\frac{1}{2} u^{2}} H_{n}(u) \\
& \times\left(\left(\sqrt{\pi} 2^{m} m !\right)^{-\frac{1}{2}} e^{i \sqrt{\beta} u y-i \frac{\beta}{2} x y-\frac{1}{2}(u-\sqrt{\beta} x)^{2}} H_{m}(u-\sqrt{\beta} x)\right) .
\end{aligned}
$$

From Eq. (5.24), we see that the wave function $\langle\xi|(x, y), \beta, m>$ defined in (5.7) has the form:

$$
<\xi \mid(x, y), \beta, m>=\left(\sqrt{\pi} 2^{m} m !\right)^{-\frac{1}{2}} e^{-i \sqrt{\beta} \xi y+i \frac{\beta}{2} x y-\frac{1}{2}(\xi-\sqrt{\beta} x)^{2}} H_{m}(\xi-\sqrt{\beta} x) .
$$

This ends the proof.

Finally, by the help of Proposition 5.1, one can use a little calculation to arrive at the announced formula:

Theorem 5.1. Let $m=0,1,2, \cdots, \beta>0$ and $a, b \in \mathbb{R}$. Then,

$$
\begin{aligned}
\sum_{k=-m}^{+\infty} \frac{(\sqrt{\beta}(a+i b))^{k}}{2^{k}(k+m) !} & L_{m}^{(k)}\left(\frac{\beta}{2}\left(a^{2}+b^{2}\right)\right) H_{k+m}(\xi) \\
= & \frac{1}{m !} \exp \left(-\frac{\beta}{4}(a-i b)^{2}+\xi(a-i b) \sqrt{\beta}\right) H_{m}(\xi-a \sqrt{\beta})
\end{aligned}
$$

Remark 5.2. As mentioned in Section 1, if we take $m=0, b=0$ and $\tau=\frac{\sqrt{\beta}}{2} a$, then Eq. (5.26) reduces to the generating function in (1.4).

Remark 5.3. According to Definition 5.2, the coherent states transform associated with the constructed coherent states have been used in [18] to characterize bound states of the planar Landau problem as integral transforms of square integrable functions on the real line. 


\section{References}

[1] Gradshteyn I.S. and Ryzhik I.M., "Table of Integrals, Series and Products", Academic Press, INC (1980)

[2] A Letter from Prof. Mourad ISMAIL, March 3, 2010 (private communication).

[3] Schrodinger E., Der stretige Ubergang von der Mikro-zur Makromechanik, Naturwissenschaften. 14, pp.664-666 (1926)

[4] Feynman R.P., An operator calculus having applications in quantum electrodynamics, Phys. Rev. 84, pp.108-128 (1951)

[5] Glauber R.J., Some notes on multiple boson processes , Phys. Rev. 84, pp.395-400 (1951)

[6] Iwata G., Non-Hermitian operators and eigenfunction expansions, Prog. Theor. Phys. 6, pp 216-226, (1951).

[7] Perelomov A., Coherent States for Arbitray Lie group, Commun. Math. Phys. 26, pp.222236 (1972)

[8] Dodonov V.V., 'Noncalssical' states in quantum optics: a 'squeezed review of the first 75 years, J.opt.B: Quantum Semiclass.opt. 4, R1-R33 (2002)

[9] Raikov G.D. and Warzel S., Quasi-Classical versus non-classical spectral asymptotics for magnetic Schrodinger operators with decreasing electric potential, Reviews in Mathematical Physics, vol 14, No.10 (2002) 1051-1072

[10] Landau L. and Lifchitz E., Quantum Mechanics: Non-relativistic Theory, New York: Pergamon (1977)

[11] Folland G.B., Harmonic analyse on phase space, in Annals of Math Studies No.122, Princeton U.P., Princeton NJ (1989)

[12] Taylor M.E., Noncommutative Harmonic Analysis, Vol 22, Mathematical Survey and monographs, AMS, Providence, RI (1986)

[13] Benson C., Jenkins J. and Ratclif G., Bounded K-spherical functions on Heisenberg groups, J.Funct. Ann, 105, pp.405-443 (1992)

[14] Duflo M. and Moore C.C., On the regular representation of a Nonunimodular locally compact group, J. Funct. Anal, 21, 2, pp.209-243 (1976)

[15] Thirulogasantar K. and Saad N.: Coherent states associated with the wavefunctions and the spectrum of the isotonic oscillator, J. Phys. A: Math. Gen. 37 (2004), 4567-4577.

[16] Titchmarsh E.C., Introduction to the theory of Fourier integrals, 2nd ed., Clarendon Press (1950)

[17] Szegö G., Orthogonal polynomials. American Mathematical Society; Providence, R.I. (1975) 
[18] Mouayn Z., Characterization of two-dimensional Euclidean Landau states by coherent state transforms, J. Phys. A : Math. Gen. 37, pp. 4813-4819 (2004) 